\title{
Intense Selections and the Speed of Evolution
}

THE Darwinian theory of organic evolution is so widely accepted that it is easy to forget that the direct evidence for it is limited to a few cases in which evolution has been studied in the wild. Perhaps the best known of these is that of the peppered moth, Biston betularia, whose black form has become abundant in Britain since the Industrial Revolution.

It had been thought that advantageous characters only slightly increased the viability of an animal; selective advantage was low, and evolution invariably slow. Recent work has shown that this assumption was unjustified. A common British butterfly of grassland, the meadow brown, Maniola jurtina, bears a series of spots on the underside of its wing. The number of spots, which is controlled by many genes, varies from none to five. A team of workers led by Ford ${ }^{1}$ discovered that the distribution of spot frequencies for female butterflies remained strikingly constant over most of England, despite great differences in climate. In south-west England, however, the spot frequency distribution was different, and a larger number of insects had two spots. At the boundary of these two areas one would have expected populations with intermediate spot-frequency distributions, and a gradual transition between the two main types of distribution over, perhaps, tens of miles. Instead, the boundary line was found to be a hedge, over which insects were flying freely.

This combination of stability over a huge area, and sudden transition to an alternative distribution, is a result of very high selection pressures. This has been confirmed by work in the Isles of Scilly, where each island is characterized by a particular spot-frequency distribution of Maniola. Great changes in spotfrequency distribution have been seen to take place within a year in association with ecological changes. On the other hand, under constant ecological conditions, when the population of Maniola was reduced to a few hundred individuals, in the next generation the population had regained not only a considerable size but also its characteristic spot distribution. The selection had been strong enough to overcome chance effects acting on the reduced population, such as the "accidental" death of insects possessing advantageous genes. It has been calculated that as many as 80 or 90 per cent of animals of certain spot-numbers may be selectively eliminated within a fow months.

It is most unlikely that this very high death rate is caused by the spots themselves. The genes which produce the spots must be responsible for more important characters. Work is going on to discover what these are ${ }^{2}$. It has been shown that animals with more spots are susceptible, in some unknown way, to the attacks of a parasitic insect, Apanteles. There is also a possibility that the bacteria in the intestines of Maniola may be concerned with the selection against certain forms.
A similar situation has recently been shown to exist in populations of a grass, Agrostis tenuis ${ }^{3,4}$. This plant has various forms characterized by their hardiness in particular climatic or chemical conditions. Some are able, for example, to survive in windy conditions, or in the face of sea spray, or where the soil concentration of metals is high. These conditions may change over a few yards, as where slag from a mine has been heaped up in a field where the soil has not been contaminated by metal. Because of the very high selective pressures acting to eliminate forms which are inviable in the particular microhabitat, adjacent small populations of Agrostis consist of very different forms, even though there is cross pollination between the two populations. In this case, then, strong disruptive selection opposes and overcomes the effects of gene flow.

Another example of rapid evolution was investigated by Dobzhansky and Pavlovsky in California ${ }^{1}$. One of the chromosomes of the fruit fly, Drosophila pseudobscura, is marked by the reversal of the genetic material at one point. Many such inversions, as they are called, appear in the chromosomes of this species, this one being known as Pike's Peak (P.P.). In the 1940s the frequency of this inversion was so low that it was effectively absent in California. Since then, however, there has been a rapid increase in the percentage of animals bearing the inversion, and a corresponding decrease in the percentage of animals bearing another important inversion. This major change in the genetic configuration of the species does not seem to have been a product of any gross meteorological change. Yet an increase in the frequency of P.P. has taken place in areas of the state with very different climates. Nor is the phenomenon associated with towns or industries; there seem to be no obvious differences between populations in country and town. Migration cannot explain the phenomenon, and it is unlikely that increased radiation levels are its cause. And it is a mystery not only why the increase came about, but how the P.P. inversion has become prevalent throughout so vast a space in so short a time.

It was long ago pointed out by Ford that evolution is most rapid, and can be most easily studied, when populations are small, when there exist two or more forms of the same species within the same habitat, or when the selective advantage of a character is great. It now appears that these conditions are fulfilled more often than had been supposed. It is therefore necessary to revise views of the speed of evolution and of the part which selection plays. Selection has always been thought to have been important, but in many eases it may be overwhelmingly so.

\footnotetext{
${ }^{1}$ References in Ford, E. B., Ecological Genetics (Methuen, 1964).

${ }^{2}$ References in Dowdeswell, W. H., and McWhirter, K., Heredity, 22, 181 (1967).

${ }^{3}$ Gregory, R. P. J., and Bradshaw, A. D., New Phytol., 64, 131 (1965)

'Jain, S. K., and Bradshaw, A. D., Heredity, 21, 407 (1966).
} 